\title{
Aplasia medular adquirida, experiencia en un hospital público de referencia
}

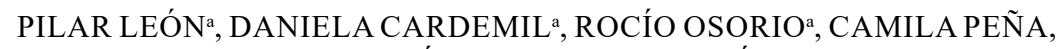
XIMENA VALLADARES, BÁRBARA PUGA, MARÍA ELENA CABRERA

\section{Acquired aplastic anemia. Experience in a public hospital}

Background: The first line treatment for patients $<40$ years old with aplastic anemia (AA) is allogeneic HLA-identical sibling donor transplantation (SCT). Immunosuppressive therapy (IST) with a combination of Thymoglobuline (ATG) and cyclosporine is used for older patients or those without a donor. Five year overall survival (OS) for both therapies is $>70 \%$. Aim: To report the experience with SCT and ATG for AA in a public hospital. Patients and Methods: AA was diagnosed in 42 patients between 1998 and 2016, according to Camitta criteria. Thirty eight (90\%) received treatment, 7 (18\%) under 40 years old received SCT, and 31 (82\%) IST. The rest were not treated. OS was calculated from date of diagnosis until last control, death or loss from follow up. Results: Complete or partial hematologic response, was obtained in $71 \%$ and $58 \%$ of cases with SCT and IS, respectively. Five year OS was 71\% and 55\% with SCT and IST, respectively. No difference in response was observed between horse and rabbit ATG. Conclusions: SCT from an HLA-identical sibling donor had a high response rate and survival. IST instead, had a lower response and survival, due to an initial high mortality rate.

(Rev Med Chile 2018; 146: 175-182)

Key words: Anemia; Aplastic; Homologous; Immunosuppression; Stem Cell Transplantation; Transplantation.

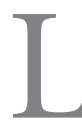
a anemia aplástica (AA) o aplasia medular adquirida es una patología infrecuente y potencialmente mortal. La incidencia en países occidentales es 2 casos por millón de habitantes ${ }^{1}$, con un peak entre 15-25 años y otro entre 65-69 años ${ }^{2}$. Se caracteriza por una disminución o ausencia de precursores hematopoyéticos en la médula ósea, que se expresa con distintos grados de citopenias en sangre periférica. Para su diagnóstico se requiere disminución de al menos dos series en sangre periférica, asociada a hipocelularidad persistente en médula ósea $(<25 \%)^{2}$.

Se ha identificado una amplia variedad de drogas, químicos, virus, enfermedades autoinmunes o embarazo como agentes etiológicos. Sin embargo, 70\% de los casos son idiopáticos. Fisiopatológicamente se postula que linfocitos T (LT)

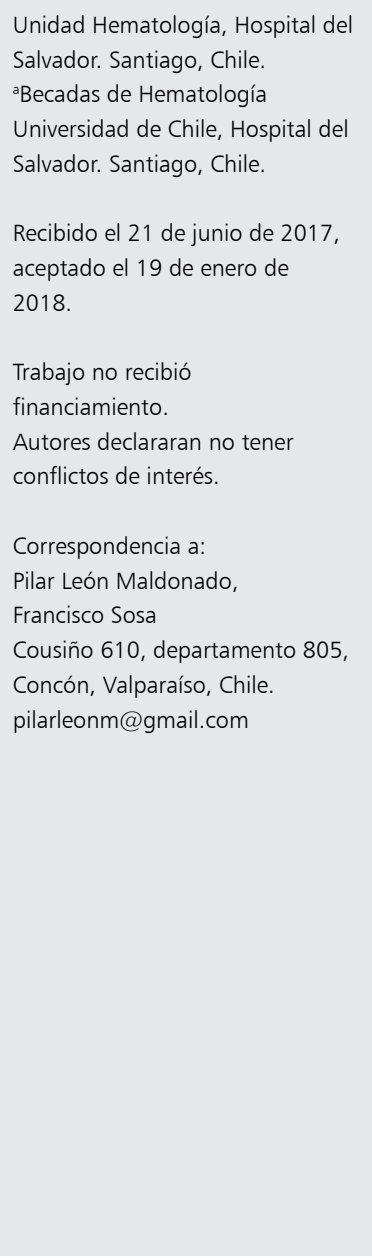

Unidad Hematología, Hospital del Salvador. Santiago, Chile. aBecadas de Hematología Universidad de Chile, Hospital del Salvador. Santiago, Chile.

Recibido el 21 de junio de 2017, aceptado el 19 de enero de 2018.

Trabajo no recibió
financiamiento.
Autores declararan no tener
conflictos de interés.
Correspondencia a:
Pilar León Maldonado,
Francisco Sosa
Cousiño 610, departamento 805,
Concón, Valparaíso, Chile.
pilarleonm@gmail.com

citotóxicos autoreactivos, mediante interferón $\gamma$ y factor de necrosis tumoral (TNF) $\alpha$, inhiben la hematopoyesis y favorecen la apoptosis de células madres hematopoyéticas CD34+ $+^{1,3}$. Las manifestaciones clínicas están asociadas a las citopenias. El diagnóstico es de exclusión, por lo que se debe descartar falla medular congénita, síndrome mielodisplástico (SMD) hipoplástico y hemoglobinuria paroxística nocturna (HPN). Alrededor de $40-50 \%$ de los pacientes con AA presentan al diagnóstico pequeños clones hematopoyéticos con ausencia de glicosilfosfatidilinositol (GPI) aunque su evolución a HPN manifiesta es infrecuente ${ }^{4}$.

El tratamiento de elección en menores de 40 años es el TPH alogénico con donante familiar idéntico (DFI), con tasa de curación superior a $70 \%{ }^{4,-8}$. El registro del Grupo Europeo de Tras- 
plante muestra sobrevida a 10 años de $86 \%, 76 \%$ y 55\%, en $<20$ años, 21-40 años y $>40$ años, respectivamente ${ }^{4}$. Las principales causas de morbimortalidad asociadas a TPH son infecciosas, enfermedad injerto vs huésped (EICH) y en $5 \%$ $15 \%$ el fallo de implante, que requiere rescate con un segundo $\mathrm{TPH}^{4,9}$.

En pacientes sin DFI o mayores de 40 años, el tratamiento inmunosupresor (TIS) con globulina antitimocítica (GAT) de caballo combinado con ciclosporina A, logra respuesta hematológica $(\mathrm{RH})$ entre $45 \%$ y $75 \%$ a los seis meses ${ }^{3,10,11}$. La sobrevida a 5 años alcanza $64 \%-76 \%{ }^{10,12}$. El registro Europeo de Trasplante también muestra que la edad es un factor pronóstico, con sobrevida a 10 años de $82 \%, 69 \%$ y $58 \%$ en $<20$ años, $21-40$ años y $>40$ años, respectivamente ${ }^{4}$. Sin embargo, el TIS, puede no ser curativo, existe riesgo de recaída, falta de respuesta y evolución clonal tardía. La recaída a 5 años varía entre 30\%-35\% asociada generalmente a discontinuación de ciclosporina $a^{9,13}$. El riesgo de desarrollo de SMD varía entre $15 \%-20 \%$ a 20 años y menos frecuente, leucemia mieloide aguda ${ }^{13}$.

Scheinberg, et $\mathrm{al}^{14}$, en un estudio randomizado, mostró superioridad de GAT de caballo en comparación con GAT de conejo en primera línea, con RH a 6 meses de $68 \%$ vs $37 \%$ y sobrevida a 3 años de $96 \%$ versus $76 \%$, respectivamente. Sin embargo, esta diferencia no ha sido corroborada

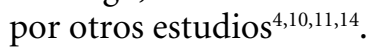

En caso de fracaso o recaída post GAT de caballo, se puede utilizar un segundo curso de inmunosupresión con Ig de conejo, o viceversa, con respuestas cercanas a $30 \%$ en fracaso a primera línea ${ }^{15,16}$ y $50 \%-60 \%$ en recaída ${ }^{4}$.

Hay escasa información en la literatura nacional sobre AA en adultos, sólo reporte de $\operatorname{casos}^{17,18,19}$.

El objetivo del presente trabajo fue describir las características epidemiológicas, clínicas, respuesta a tratamiento y sobrevida, de pacientes con diagnóstico de aplasia medular severa (AMS) y aplasia medular muy severa (AMMS) tratados en el Hospital del Salvador.

\section{Materiales y Métodos}

\section{Pacientes}

Estudio descriptivo de una serie de pacientes mayores de 15 años, con AMS y AMMS, diagnóstico realizado entre enero de 1998 y diciembre de 2016, en el Servicio de Medicina, Sección He- matología del Hospital del Salvador (Centro de Referencia de la I, II y III Regiones y Área Oriente de la Región Metropolitana).

\section{Epidemiología, clínica y laboratorio}

Se revisaron fichas clínicas y se registraron antecedentes clínicos y de laboratorio como edad al diagnóstico, género, comorbilidades, sintomatología, hemograma, antígeno de superficie para virus hepatitis $\mathrm{B}(\mathrm{VHB})$, anticuerpo antihepatitis $\mathrm{C}$, antígeno virus de inmunodeficiencia humana (VIH), serología para citomegalovirus (CMV), virus Epstein-Barr (VEB), parvovirus B-19, autoanticuerpos, citometría de flujo para descartar hemoglobinuria paroxística nocturna (HPN), mielograma, cariograma, biopsia de médula ósea y estudio HLA de los hermanos, en $<40$ años.

\section{Severidad de la aplasia}

Se documentó la severidad de la enfermedad, clasificando los pacientes como AMS con dos de los siguientes criterios (Criterios de Camitta ${ }^{20,21}$ ): 1) recuento absoluto de neutrófilos $(\mathrm{RAN})<500 /$ $\mathrm{ul}, 2$ ) recuento de plaquetas $<20.000 /$ ul y recuento de reticulocitos corregido $<1 \%$. Se usó la definición de AMMS si el RAN fue $<200 /$ ul $^{2}$.

\section{Tratamiento}

Los pacientes $<40$ años con DFI recibieron $\mathrm{TPH}$ con acondicionamiento inmunosupresor con GAT de caballo o conejo, según disponibilidad del fármaco en nuestro país y ciclofosfamida 50 $\mathrm{mg} / \mathrm{kg}$ por 4 días. Para prevenir el rechazo de injerto contra huésped (EICH), se utilizó ciclosporina en dosis de $5 \mathrm{mg} / \mathrm{kg} /$ día oral, ajustado para mantener nivel entre 200-350 ng/ml. La ciclosporina se mantuvo al menos 12 meses y luego se redujo lentamente para evitar el rechazo.

Los $>40$ años y menores sin DFI, recibieron TIS con GAT de caballo (Merieux) hasta 2007, $15 \mathrm{mg} / \mathrm{kg} / \mathrm{d}$ infusión intravenoso (iv) en 12-18 h por 5 días y desde 2008 (ATGAM, Pfeizer) 40 $\mathrm{mg} / \mathrm{Kg}$ iv por 4 días, o de conejo (Timoglobulina, Genzyme) 3,5 mg/kg infusión iv por 5 días, según disponibilidad, asociado a ciclosporina en dosis de $5 \mathrm{mg} / \mathrm{kg} /$ día oral (200-400 mg/día) ajustado para mantener nivel entre $200-350 \mathrm{ng} / \mathrm{ml}$. La ciclosporina se mantuvo al menos 12 meses y luego se redujo lentamente para evitar recaídas.

Todos los pacientes recibieron transfusión profiláctica de plaquetas para mantener recuento 
plaquetario $>10.000 / \mathrm{ul}(\mathrm{o}>20.000 /$ ul en presencia de fiebre), transfusión de glóbulos rojos para mantener hemoglobina $>7 \mathrm{~g} / \mathrm{dl}$ y tratamiento antibiótico con evidencia de fiebre y/o signos de infección.

\section{Respuesta}

La respuesta se evaluó a los 6 meses y se consideró completa (RC) si: $\mathrm{Hb}>10 \mathrm{gr} / \mathrm{dl}$, RAN $>1.500 /$ ul y plaquetas $>100.000 /$ ul; parcial (RP) si: RAN $>500 /$ ul y plaquetas $>20.000 /$ ul y refractaria (R) con valores menores ${ }^{4,20}$. Los pacientes refractarios fueron sometidos a un segundo curso de inmunosupresión.

\section{Análisis estadístico}

La sobrevida global (SG) se calculó desde la fecha de diagnóstico hasta el último control, abandono o muerte, usando el método de Kaplan-Meyer y las comparaciones a través del test long-rank.

El estudio contó con aprobación por el Comité de Ética del Servicio de Salud Metropolitano Oriente.

\section{Resultados}

Cuarenta y siete pacientes cumplieron criterio diagnóstico de AA severa o muy severa y se excluyó cinco por falta de datos.

Las características clínicas de los 42 pacientes evaluados se presentan en la Tabla 1. La mediana de edad al diagnóstico fue 30 años (rango 15-84), con un peak de incidencia entre 15-20 años. El 40\% (17/42) fue mayor de 40 años; relación hombre/ mujer: $1 / 1,5$.

Dos tercios se presentaron como AMS y un tercio como AMMS. Cinco pacientes contaban con antecedente de exposición a tóxicos (pesticidas 2 casos), y drogas de uso recreativo (pasta base de cocaína 2 casos y cocaína 1 caso), tres con antecedente de hepatitis seronegativa previa al diagnóstico, dos embarazo/puerperio, dos fármacos (dipirona y fenitoína) y uno enfermedad autoinmune. En 29 pacientes (69\%) no se encontró causa, siendo catalogados como AA idiopática. La serología para VIH y VHB fue negativa en todos los pacientes y positiva para VHC en un paciente. En 53\% (18/34) de los casos estudiados se detectó un clon pequeño de HPN por citometría de flujo al diagnóstico. En $1 / 22$ casos con estudio citogenético, se detectó una alteración constitucional (Síndrome de Klinefelter) y en el resto fue normal. La biopsia de médula ósea (MO) mostró una celularidad $<25 \%$ en todos los pacientes.

De los 42 casos evaluables, tres recibieron ciclosporina solamente, por falta de GAT, uno de los cuales obtuvo respuesta completa después de un año. El otro no recibió tratamiento por fallecimiento por infección al mes del diagnóstico.

De los 38 pacientes que recibieron tratamiento según protocolo, en 7 (18\%) se realizó TPH con DFI y en 31 (82\%) se administró TIS con GAT de caballo o conejo.

En el grupo sometido a TPH con DFI, 5/7 (71\%) pacientes presentaron respuesta hematológica a los 6 meses (4 RC y $1 \mathrm{RP}$ ), un paciente falleció por una hemorragia intracerebral al segundo mes del trasplante y otro presentó una pérdida del injerto al cuarto mes, requiriendo un segundo trasplante y falleciendo por EICH agudo.

En el grupo sometido a TIS, 16/31 (51,6\%) pacientes presentaron respuesta hematológica $(7$ RC y 9 RP), 9/31 (29\%) fueron refractarios y 6/31 (19\%) fallecieron antes de documentar la respuesta, cinco por infección y uno por hemorragia.

De los 9 pacientes refractarios, cuatro recibieron una segunda dosis de GAT (dos con respuesta hematológica y dos fallecieron sin respuesta), dos

Tabla 1. Características clínicas de pacientes adultos ( $n=42$ ) con aplasia medular adquirida, Hospital del Salvador, Chile

\begin{tabular}{|ll|}
\hline Variables & n (\%) \\
\hline Edad (años), mediana (rango) & $30(15-84)$ \\
$\quad \leq 40$ años & $25(60 \%)$ \\
$\quad>40$ años & $17(40 \%)$ \\
Sexo (hombre/mujer) & $17 / 25$ \\
\hline Severidad AMS/AMMS & $28(67 \%) / 14(33 \%)$ \\
\hline Sangrado muco-cutáneo al ingreso & $37(88 \%)$ \\
\hline Sind. anémico al ingreso & $31(74 \%)$ \\
\hline Infección al ingreso & $12(29 \%)$ \\
\hline Hemoglobina (gr/dl) & $5,7(3,0-10,8)$ \\
\hline Neutrófilos (ul) & $500(7-4.500)$ \\
\hline Plaquetas (ul) & $7.000(1.700-38.000)$ \\
\hline Idiopática & $29(69 \%)$ \\
\hline Pequeño clon HPN & $18 / 34(53 \%)$ \\
\hline Citogenética normal & $21 / 22(95 \%)$ \\
\hline
\end{tabular}

AMS = Aplasia medular severa; $\mathrm{AMMS}=$ aplasia medular muy severa; HPN= Hemoglobinuria paroxística nocturna. 
Tabla 2. Características y resultados de pacientes adultos $(n=38)$ con aplasia medular adquirida, tratados en Hospital del Salvador, Chile

\begin{tabular}{|c|c|c|c|}
\hline Sexo/edad & Tratamiento & RH 6 meses & Resultado/tiempo seguimiento \\
\hline 1.- $\mathrm{H} / 32$ & $\mathrm{TPH}$ & $\mathrm{RHC}$ & Vivo, abandona controles 12 meses \\
\hline 2.- $M / 19$ & $\mathrm{TPH}$ & $\mathrm{RHC}$ & Vivo, en remisión / 80 meses \\
\hline 3.- $M / 17$ & $\mathrm{TPH}$ & $\mathrm{RHC}$ & Vivo, en remisión / 58 meses \\
\hline 4.- $M / 20$ & $\mathrm{TPH}$ & $\mathrm{RHC}$ & Vivo, en remisión / 83 meses \\
\hline 5.- $M / 24$ & $\mathrm{TPH}$ & REF & Fallece, EICH agudo 2do TPH / 9 meses \\
\hline 6.- $M / 16$ & $\mathrm{TPH}$ & RHP & Vivo, en remisión/ 32 meses \\
\hline 7.- $M / 15$ & $\mathrm{TPH}$ & Fallece & Fallece, hemorragia intracerebral / 5 meses \\
\hline 8.- $\mathrm{H} / 19$ & TIS Caballo & REF & Vivo, logra RHP, en remisión / 139 meses \\
\hline 9.- $M / 49$ & TIS Conejo & $\mathrm{RHC}$ & Vivo, en remisión / 89 meses \\
\hline 10.- $H / 29$ & TIS Conejo & $\mathrm{RHC}$ & Vivo, en remisión / 118 meses \\
\hline 11.- $\mathrm{H} / 23$ & TIS Conejo & REF & Fallece, rechaza 2 da dosis TIS / 56 meses \\
\hline 12.- $M / 42$ & TIS Caballo & REF & Fallece, abandona controles / 32 meses \\
\hline 13.- H/51 & TIS Conejo & RHP & Fallece, recaída 2do TIS / 17 meses \\
\hline 14.- $M / 54$ & TIS Conejo & REF & Vivo, 2do TIS, RHP / 40 meses \\
\hline 15.- $M / 31$ & TIS Conejo & RHP & Vivo, en remisión/ 39 meses \\
\hline 16. $-H / 28$ & TIS Conejo & REF & Fallece, 2do TIS, TPH DFI / 137 meses \\
\hline 17.- $\mathrm{H} / 15$ & TIS Conejo & REF & Fallece, 2do TIS / 21 meses \\
\hline 18.- $M / 15$ & TIS Caballo & RHP & Vivo, en remisión/ 54 meses \\
\hline 19.- $M / 68$ & TIS Conejo & REF & Fallece, pancreatitis aguda / 13 meses \\
\hline 20.- $\mathrm{H} / 23$ & TIS Conejo & REF & Vivo, 2do TIS, TPH DFI, en remisión / 90 meses \\
\hline 21.- $M / 60$ & TIS Conejo & RHP & Vivo, en remisión / 123 meses \\
\hline 22.- $M / 48$ & TIS Conejo & $\mathrm{RHC}$ & Vivo, abandona controles / 3 meses \\
\hline 23.- $M / 63$ & TIS Conejo & RHP & Vivo, recaída 2do TIS, en remisión / 106 meses \\
\hline 24.- $M / 50$ & TIS Conejo & RHP & Vivo, en remisión / 36 meses \\
\hline 25.- $M / 17$ & TIS Caballo & REF & Vivo, esperando 2do TIS / 15 meses \\
\hline 26.- $M / 41$ & TIS Caballo & Fallece & Fallece, abandona controles / 3 meses \\
\hline 27.- $M / 26$ & TIS Caballo & REF & Vivo, logra RHP / 25 meses \\
\hline 28.- $M / 37$ & TIS Caballo & $\mathrm{RHC}$ & Vivo, en remisión / 22 meses \\
\hline 29.- $M / 21$ & TIS Caballo & Fallece & Fallece / 2 meses \\
\hline 30.- $\mathrm{H} / 20$ & TIS Caballo & $\mathrm{RHC}$ & Vivo, abandona controles / 3 meses \\
\hline 31.- $\mathrm{H} / 27$ & TIS Caballo & $\mathrm{RHC}$ & Vivo, en remisión / 15 meses \\
\hline 32.- $M / 44$ & TIS Conejo & Fallece & Fallece / 6 meses \\
\hline 33.- $M / 67$ & TIS Conejo & Fallece & Fallece / 1 mes \\
\hline 34.- $\mathrm{H} / 43$ & TIS Caballo & $\mathrm{RHC}$ & Vivo, en remisión/ 6 meses \\
\hline 35.- H/65 & TIS Caballo & REF & Fallece / 7 meses \\
\hline 36.- H/56 & TIS Conejo & Fallece & Fallece / 2 meses \\
\hline 37.- $H / 62$ & TIS Caballo & RHP & Vivo, 2do TIS, en remisión / 228 meses \\
\hline 38.- H/17 & TIS Conejo & Fallece & Fallece / 5 meses \\
\hline
\end{tabular}

$\mathrm{TPH}=$ Trasplante de progenitores hematopoyéticos; $\mathrm{TIS}=$ Tratamiento inmunosupresor; $\mathrm{RH}=$ Respuesta hematológica; $\mathrm{RHC}=$ Respuesta hematológica completa; $\mathrm{RHP}=$ Respuesta hematológica parcial; REF = Refractario. 
abandonaron control, dos fallecieron antes de recibir la segunda dosis y uno se encuentra en espera de recibirlo. En suma, 18/31 (58\%) pacientes respondió al TIS, 16 con la primera infusión y dos en una segunda oportunidad.

$\mathrm{Al}$ analizar el grupo tratado con inmunosupresión, 18/31 (58\%) recibió GAT de conejo y el resto de caballo, según disponibilidad del fármaco. De los pacientes que recibieron globulina de conejo, 8/18 (44\%) presentaron respuesta hematológica (RC/RP) a los 6 meses, 6/18 (33\%) fueron refractarios y $4 / 18(22 \%)$ fallecieron antes de documentar respuesta. De los pacientes que recibieron globu- lina de caballo, 6/13 (46\%) presentaron $\mathrm{RC} / \mathrm{RP}$ a los 6 meses, $5 / 13$ (39\%) fueron refractarios y $2 / 13$ (15\%) falleció antes de documentar respuesta.

La mediana de seguimiento fue 24 meses (rango 1-228). La SG a 5 años del grupo tratado con TPH DFI fue $71 \%$ y del grupo tratado con TIS fue 53\% $(\mathrm{p}=0,57)$, Figura 1. La SG a 5 años de los pacientes que recibieron GAT de conejo fue $50 \%$ y de caballo 56\% ( $p=0,49)$. Respecto a la severidad de la aplasia, la SG a 5 años en AAMS fue 59\% y en AMS $55 \%(\mathrm{p}=0,73)$, y respecto a la edad, la sobrevida en $\leq 40$ y $>40$ años, fue $65 \%$ y $44 \%$, respectivamente $(\mathrm{p}=0,19)$, Figura 2.
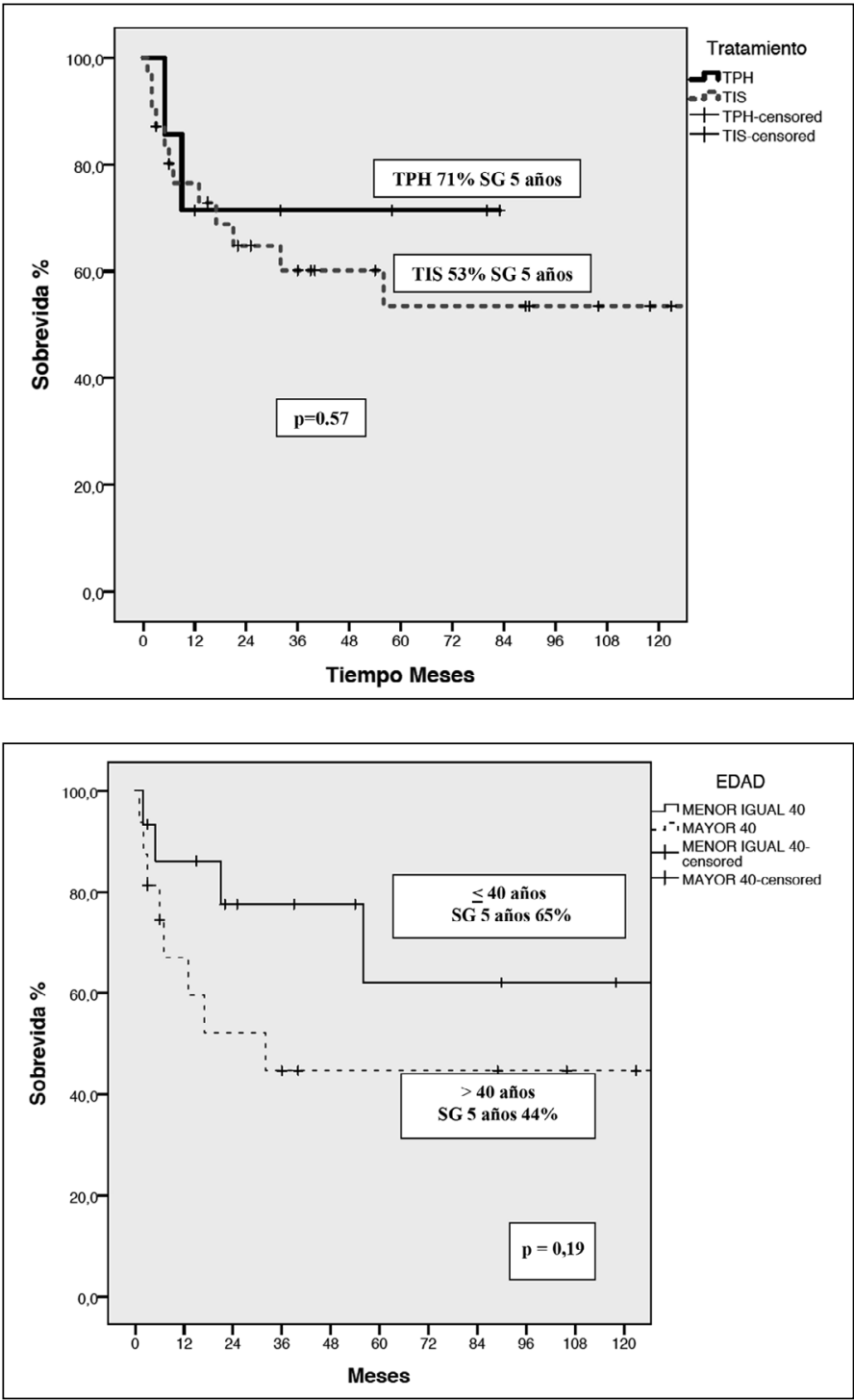

Figura 1. Sobrevida de pacientes con aplasia medular adquirida, según tipo de tratamiento. Curva de Kaplan Meier.
Figura 2. Sobrevida de pacientes con aplasia medular adquirida, tratados con TIS, según grupo etario. Curva de Kaplan Meier. 


\section{Discusión}

Se presenta una serie de pacientes adultos chilenos con diagnóstico de AAS o AAMS, tratados en el Hospital del Salvador.

Los resultados son similares a la literatura respecto a aquellos que recibieron TPH con DFI, con SG $>70 \%$ a 5 años. No es posible evaluar la mortalidad relacionada a trasplante, por el bajo número de casos. Los resultados con TIS fueron inferiores a lo reportado, con rangos que varían en RH entre 60\%-70\% y SG a 10 años entre 58\%$82 \%$, según el grupo etario, en comparación con RH de $58 \%$ y SG a 5 años de 53\%, observada en nuestra casuística. Una explicación a esta diferencia, con el TIS, se debe probablemente a la elevada mortalidad en los primeros 6 meses (19\%) por infecciones, antes de lograr la recuperación hematológica y a pesar del apoyo de antimicrobianos. El uso de una segunda dosis de inmunosupresión se justifica, ya que la mitad de ellos respondió y debiera indicarse precozmente, si no se observa recuperación hematológica a los 3 meses. A nivel nacional, destacan dos publicaciones de series de casos en pacientes pediátricos ${ }^{23,24}$. Tordecilla et al y Barriga et al., con 7 y 25 niños, respectivamente, 8 casos tratados con TPH y 22 casos con TIS. La sobrevida a 5 años descrita para los pacientes que recibieron TPH fue $71 \%$ y $79 \%$, respectivamente, similar a lo descrito en estudios pediátricos internacionales. La RH al tratamiento inmunosupresor también fue baja, 50\%, probablemente relacionado a la administración más tardía de tratamiento inmunosupresor, por la dificultad de acceso que ha existido de estos medicamentos. Cabrera et $\mathrm{al}^{17}$ publicó un reporte de dos adultos tratados en el Hospital del Salvador con TIS, alcanzando ambos respuesta hematológica completa y sobrevida mayor a 10 años.

El peak de incidencia se observó en pacientes jóvenes, tal como está descrito.

Igualmente, en la mayoría de los casos, dos trcios, fueron catalogados de causa idiopática ${ }^{22}$. En nuestra serie, dentro de las causas probables más frecuentes se encontraron las drogas recreativas, como pasta base de cocaína y cocaína, especialmente en jóvenes. Se ha descrito reporte de casos de AAMS con el uso de éxtasis (metanfetamina sintética ${ }^{25}$, pero el uso de pasta base de cocaína que contiene solventes orgánicos como querosene y además sustancias alquilantes, monóxido de carbono, ácido sulfúrico entre otros ${ }^{26}$ es una droga utilizada en países latinoamericanos por su bajo costo, no ha sido reportada como causa de AA. En la mitad de nuestros casos, se detectó un clon de HPN, cifra concordante con la literatura ${ }^{27-30}$. Estos clones son pequeños y no producen sintomatología específica. En cuanto a su significado pronóstico, algunos estudios ${ }^{30}$ sugieren que estos pacientes responderían mejor al tratamiento inmunosupresor. Sin embargo, no hay consenso al respecto.

El estudio citogenético es de utilidad para descartar alteraciones clonales, como mielodisplasia variante hipocelular. Generalmente es normal, pero puede haber anormalidades citogenéticas en $6 \%-10 \%$ de los casos $^{31}$. En nuestro estudio, la cifra fue menor, $4,5 \%$ y solo una alteración constitucional. Una razón podría estar en la dificultad de obtener metafases en muestras con escasa celularidad.

No hay duda de que, el TPH DFI es el tratamiento de elección en primera línea para pacientes menores de 40 años, con excelente expectativa de vida ${ }^{4,5}$. Sin embargo, menos de un tercio de pacientes tiene un $\mathrm{DFI}^{8}$. Las últimas guías del Grupo Europeo de Trasplante recomiendan una primera dosis de TIS como primera línea en caso de pacientes mayores de 40 años, o que no cuenten con DFI. De no observarse respuesta a los 6 meses se debiera proceder a un trasplante con donante alternativo, que puede ser un donante no relacionado o de cordón umbilical ${ }^{4}$. La sobrevida con estos trasplantes ha mejorado, llegando a cifras comparables a las de un DFI, aunque con mayor riesgo de EICH. El trasplante con donante haploidéntico, se considera aún experimental ${ }^{32}$.

En cuanto al tratamiento inmunosupresor, ha habido discontinuidad de abastecimiento en nuestro país. Por varios años se dispuso sólo GAT de conejo o sólo de caballo y en los últimos 8 años, de ambos. Esta dificultad, el retraso en el inicio de la terapia, podría explicar en parte los bajos resultados observados en este grupo de pacientes (SG a 5 años 53\%).

Uno de los desafíos actuales es garantizar el acceso inmediato a TIS y el uso de una segunda dosis a los 3 a 6 meses en caso de refractariedad, de la otra variedad de GAT.

La optimización de las medidas de soporte durante el período de aplasia, es otro punto importante a considerar, como la hospitalización en 
unidades de aislamiento con personal especializado, el soporte antibiótico y transfusional. Es muy probable que los primeros pacientes tratados con TIS de nuestra serie, recibieron un menor soporte que los más recientes, como el ingreso a unidades de aislamiento, o de paciente crítico, apoyo infectológico, entre otras medidas de apoyo. Estas falencias podrían explicar la mortalidad observada. Además, en 2014 se aprobó el uso de Eltrombopag, agonista sintético del receptor de trombopoyetina, en pacientes refractarios ${ }^{33}$ que mejora la trombocitopenia y actualmente se evalúa su utilidad en primera línea asociado a TIS ${ }^{4}$.

\section{Conclusión}

Los resultados observados en nuestro estudio en AA del adulto que reciben tratamiento con TPH son comparables a la literatura, no así en el grupo tratado con inmunosupresión. Esperamos que un tratamiento inmunosupresor oportuno, y las medidas de soporte estandarizadas, en uso actualmente en nuestro hospital, permitan mejorar los resultados en el grupo de pacientes que no tienen acceso a TPH en primera línea.

\section{Referencias}

1. Young N, Calado R, Scheinberg P. Current concepts in the pathophysiology and treatment of aplastic anemia. Blood 2006; 108: 2509-19.

2. Brodsky R, Jones R. Aplastic anaemia. Lancet 2005; 365: 1647-56.

3. Young N. Current concepts in the pathophysiology and treatment of aplastic anemia. Educational Program American Society of Hematology 2016.

4. Bacigalupo A. How I treat acquired aplastic anemia. Blood 2017; 129 (11): 1428-36.

5. Marsh JC, Ball SE, Cavenagh J, Darbyshire P, Dokal I, Gordon-Smith EC, et al. Guidelines for the diagnosis and management of aplastic anaemia. Br J Haematol 2009; 147: 43-70.

6. Storb R, Blume KG, O’Donnell MR, Chauncey T, Forman SF, Deeg HJ, et al. Cyclophosphamide and Antithymocyte Globulin to Condition Patients With Aplastic Anemia for Allogeneic Marrow Transplantations: The Experience in Four Centers. Biology of Blood and Marrow Transplantation 2001; 7: 39-44.

7. Armand P, Antin JH. Allogeneic Stem Cell Transplanta- tion for Aplastic Anemia. Biology of Blood and Marrow Transplantation 2007; 13: 505-16.

8. DeZern AE and Guinan EC. Therapy for Aplastic Anemia. American Society of Hematology 2011, pag 82-3.

9. Peffault de Latour R. Transplantation for bone marrow failure: current issues. Education Program American Society of Hematology 2016.

10. Afable MG, Shaik M, Sugimoto Y, Elson P, Clemente M, Makishima $\mathrm{H}$, et al. Efficacy of rabbit anti-thymocyte globulin in severe aplastic anemia. Haematologica 2011; 96 (9): 1269-75.

11. Vallejo C, Montesinos P, Polo M, Cuevas B, Morado M, Rosell A, et al. Bone Marrow Failure Spanish Study Group (Pethema-GETH). Rabbit antithymocyte globulin versus horse antithymocyte globulin for treatment of acquired aplastic anemia: a retrospective analysis. Ann Hematol 2015; 94 (6): 947-54.

12. Vaht K, Göransson M, Carlson K, Isaksson C, Lenhoff $\mathrm{S}$, Sandstedt A, et al. Incidence and Outcome in Aplastic Anemia Diagnosed 2000-2011. A Nationwide Swedish Registry Study. Blood 2016; 128: 3905.

13. Townsley D, Winkler T. Non-transplant therapy for bone marrow failure. Educations Program American Society of Hematology 2016.

14. Scheinberg P, Nunez O, Weinstein B, et al. Horse versus rabbit antithymocyte globulin in acquired aplastic anemia. N Engl J Med 2011; 365 (5): 430-8.

15. Scheinberg P, Nunez O, Young NS. Retreatment with rabbit anti-thymocyte globulin and ciclosporin for patients with relapsed or refractory severe aplastic anaemia. Br J Haematol 2006; 133 (6): 622-7.

16. Scheinberg P, Townsley D, Dumitriu B, et al. Horse antithymocyte globulin as salvage therapy after rabbit antithymocyte globulin for severe aplastic anemia. Am J Hematol 2014; 89: 467-9.

17. Cabrera ME, Pinto D. Anemia aplástica adquirida del adulto. Su tratamiento a propósito de dos casos. Rev Med Chile 2003; 131: 1439-43.

18. Soto A, Lavados M, Araya F. Rash y anemia aplásica inducidos por fenitoína: caso clínico. Rev Chil Neuro-psiquiatr 2011; 49: 171-6.

19. Ruiz C, Monroy T, Montaña N, Lora P, Lamich R, Valderrama A. Aplasia medular asociada al uso de ticlopidina: Report of one case. Rev Med Chile 2002; 130: 798-802.

20. Camitta BM, Storb R, Thomas ED. Aplastic anemia (first of two parts): pathogenesis, diagnosis, treatment, and prognosis. N Engl J Med 1982; 306: 645-52.

21. Camitta BM, Storb R, Thomas ED. Aplastic anemia (second of two parts): pathogenesis, diagnosis, treatment, and prognosis. N Engl J Med 1982; 306: 712-8. 
22. Scheinberg P, Young NS. How I treat acquired aplastic anemia. Blood 2012; 120: 1185-96.

23. Tordecilla J, Campbell M, Joannon P, Ávila R, Soto V, Rizzardini C. Anemia Aplástica: Experiencia con 7 casos. Rev Chil Pediatr 2003; 74: 179-86.

24. Barriga F, Wietstruck A, Becker A, Zúñiga P, Besa de la $\mathrm{CP}$, Álvarez M, et al. Tratamiento de anemia aplásica severa adquirida en pacientes pediátricos con inmunosupresión y trasplante alogénico de precursores hematopoiéticos. Rev Med Chile 2007; 135: 1421-8.

25. Clark AD, Butt N. Ecstasy-induced very severe aplastic anaemia complicated by invasive pulmonary mucormycosis treated with allogeneic peripheral blood progenitor cell transplant. Clinicaland Laboratory Haematology 1997; 19: 279-81.

26. www.senda.gob.cl. Prevención, tratamiento y rehabilitación. Pasta base.

27. Schrezenmeier H, Hertenstein B, Wagner B, Raghavachar A, Heimpel H. A pathogenetic link between aplastic anemia and paroxysmal nocturnal hemoglobinuria is suggested by a high frequency of aplastic anemia patients with a deficiency of phosphatidylinositol glycan anchored proteins. Exp Hematol 1995; 23: 81-7.

28. Scheinberg P, Marte M, Nunez O, Young NS. Paroxys- mal nocturnal hemoglobinuria clones in severe aplastic anemia patients treated with horse anti-thymocyte globulin plus cyclosporine. Haematologica 2000; 95 : 1075-80.

29. Sugimori C, Chuhjo T, Feng X, et al. Minor population of CD55-CD59-blood cells predicts response to immunosuppressive therapy and prognosis in patients with aplastic anemia. Blood 2006; 107: 1308-14.

30. Kulagin A, Lisukov I, Ivanova $M$, et al. Prognostic value of paroxysmal nocturnal haemoglobinuria clone presence in aplastic anaemia patients treated with combined immunosuppression: results of two-centre prospective study. Br J Haematol 2014; 164: 546-54.

31. Keung YK, Pettenati MJ, Cruz JM, Powell BL, Woodruff $\mathrm{RD}$, Buss DH. Bone marrow cytogenetic abnormalities of aplastic anemia. Am J Hematol. 2001; 66 (3): 167-71.

32. Bacigalupo A, Sica S. Alternative donor transplants for severe aplastic anemia: current experience. Seminars Hematol 2016; 53 (2): 115-9.

33. Lengline E, Drenou B, Peterlin P, Tournilhac O, Abraham J, Berceanu A, et al. Nationwide Survey on the Use of Eltrombopag in Patients with Severe Aplastic Anemia: Report on Behalf of the French Reference Center for Aplastic Anemia. Blood 2016; 128: 2684. 